\title{
BRAIN TUMOR DETECTION FROM MRI USING ADAPTIVE THRESHOLDING AND HISTOGRAM BASED TECHNIQUES
}

\author{
E. MURALI*AND K. MEENA ${ }^{\dagger}$
}

\begin{abstract}
This paper depicts a computerized framework that can distinguish brain tumor and investigate the diverse highlights of the tumor. Brain tumor segmentation means to isolated the unique tumor tissues, for example, active cells, edema and necrotic center from ordinary mind tissues of WM, GM, and CSF. However, manual segmentation in magnetic resonance data is a timeconsuming task. We present a method of automatic tumor segmentation in magnetic resonance images which consists of several steps. The recommended framework is helped by image processing based technique that gives improved precision rate of the cerebrum tumor location along with the computation of tumor measure. In this paper, the location of brain tumor from MRI is recognized utilizing adaptive thresholding with a level set and a morphological procedure with histogram. Automatic brain tumor stage is performed by using ensemble classification. Such phase classifies brain images into tumor and non-tumors using Feed Forwarded Artificial neural network based classifier. For test investigation, continuous MRI images gathered from 200 people are utilized. The rate of fruitful discovery through the proposed procedure is 97.32 percentage accurate.
\end{abstract}

Key words: MRI, Morphological, Thresholding, Brain tumor, Level set, Histogram

AMS subject classifications. 92B20, 68U10

1. Introduction. One of the necessary steps in most of the medical imaging analysis is to extract the boundary of a locality of our interest. Precise location is vital in cerebrum tumor identification. The level of exactness can be expanded through the use of computer aided system. Such framework can assist the radiologist with detecting brain tumor all the more properly. Magnetic resonance image have been extensively approved for imaging because of its competences are generating precise brain scan in less interval time, with changing contrasts, such as T1-weighted, T2-weighted and Flair. Respectively difference yields intensity dissimilarities in MRI scans. Cerebrum is a 3-D composite structure. Numerous views of brain images are axial, sagittal and coronal. In axial view the brain image was divided by a horizontal plane. The sagittal view, the brain image is divided into right and left part of the median plane and in coronal view, the brain is divided into ventral and dorsal by frontal plane. Figure 1.1 shows views of MRI brain scan.

Image enhancement techniques are used to develop the image feature for human perception. They are defined as methods of image processing such that, the result is more appropriate than the original image. Histogram equalization is a fundamental tool in image enhancement. It is likely to aid in perceptive of how they function on digital images. The equalization of intensity level method is an enhanced image with dynamic range, which will tend to have high difference. The increased in contrast is owing to the circumstance that the average intensity level of the equalized image is enhanced by the original. Overall, the increased in intensity is due to the reality that the average intensity level in the equalized image histogram is better than the original.

1.1. Related work. Mouli Laha [1] introduced an approach that embedded iterative thresholding in parallel to Otsu's global threshold. There is no need to set any limit value before the procedure. Similarly, the cropping skull stripped techniques from brain scan is efficient for three views. This method operates very well in the case of $2 \mathrm{D}$.

K Sudharani and T.C Sarma [2] proposed an algorithm that calculates the threshold qualitatively and quantitatively by methods of the standard images. It diminishes misclassification errors where the insignificant

\footnotetext{
* Research Scholar, Vel Tech Rangarajan Dr.Sagunthala R\&D Institute of Science and Technology, Avadi, Chennai, India. India

$\dagger$ CSE Department, Professor, Vel Tech Rangarajan Dr.Sagunthala R\&D Institute of Science and Technology, Avadi, Chennai,
} 


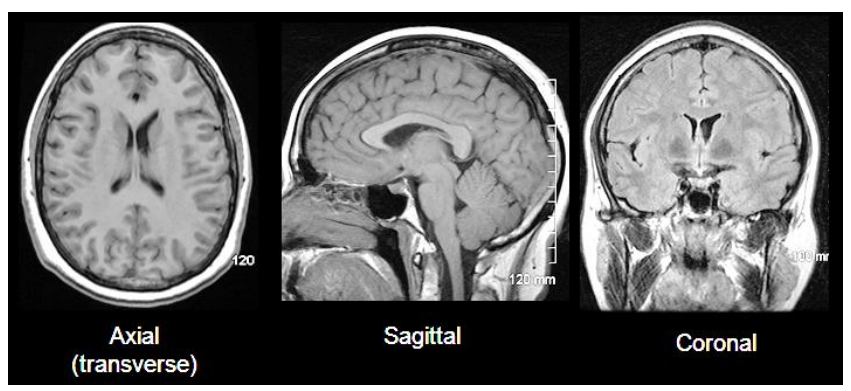

FIG. 1.1. Different angle Views of the Brain.

disparity inside each object by its own can't ensure the required result. Sudipta Roy, Bhattacharyya D [3] suggested method is an automatic, multi-scale, brain tissue segmentation algorithm that reaches to very good acceptable result. Proposed methodology has the ability to detect irregularity in the brain lesion if any. In that paper, they proposed a level set-based minimization system for the variation segmentation method by iterative perceptions and mutable the speed function of level set.

Christopher Bowels, Chen Qin [4] proposed a model for brain tissues segmentation by using an image fusion algorithm, irrespective of primary pathology. They show that an seemingly good FLAIR image can be fused from the T1-weighted image of a subject, and the distinctions among this fake FLAIR and the true FLAIR can be coupled with validity from the true FLAIR to indicate tissue location. The drawback of the proposed model is that is needs both FLAIR and T1-weighted images and any important variations in T1-weighted gaining proprieties may negatively impact on performance. The researchers isolate the images into n- scale layers with completely separate visual categories [5] to address the linguistic gap between low level and high level features. For each layer, FDA was conducted and a multi scale histogram was finally constructed. Finally, the SVM classifier is used to classify. Using Gaussian function and down sampling, each image is divided into multiscale. By using Gaussian pyramid, several distinctive histograms are constructed. The accuracy of the visual dictionary has been improved by extracting spatial features using sparse coding model. In the classification of medical images, non-negative scanty coding is performed to address the issue of optimization.

1.2. Proposed methodology. Figure 1.2 depicts the overall approach of the suggested system. In the frequency domain, smoothing is achieved by decreasing the parts of elevated frequency. The fundamental filtering model is

$$
G(u, v)=H(u, v) \cdot F(u, v)
$$

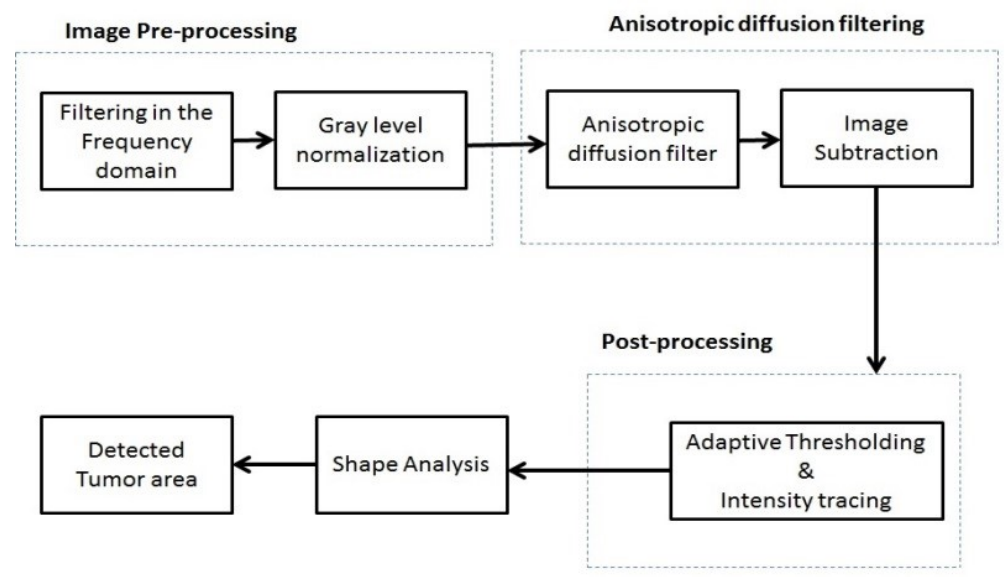

FIG. 1.2. Flow Diagram 
The image was filtered with Fourier transform $F(u, v)$ and filtered function is $H(u, v)$. From that point onward, gray level normalization is connected to change the scope of the pixel intensity. By utilizing anisotropic diffusion filter to diminish the image noise without evacuating critical parts of the image content, commonly edges, lines or different subtleties that is vital for the elucidation of the image. By using imsubtract, features are subtract from one image from another image, or to subtract a constant value from an image. Imsubtract subtracts each pixel value from the comparing pixel in the other input image in one of the input image and returns the result in the related pixel in the output image.

In adaptive thresholding modifications the threshold is dynamically over the image. Thresholding commonly takes a grayscale or colour image as an input and, in the least complex usage, yields a binary image speaking to the segmentation. The process of shape analysis consists of two main steps: (1) the extraction of image components of the target (e.g., area, boundary, network pattern and skeleton), (2) the description of the shape features (e.g., size, perimeter, circularity and compactness) and finally show the detected tumor area.

1.3. Organization of the paper. This paper is organized as follows. Section 1 describes the introduction, literature review and block diagram of the proposed method. Section 2 shows the various methodologies such as Histogram Method, Adaptive Threshold, Anisotropic Diffusion filtering and Level set method in detail. Section 3 presents the experimental results and discussion. Section 4 concludes the paper.

2. Methodology. The algorithm introduced in this section is performed by an automatic thresholding method in its place of physically changing the threshold for each image. The threshold range is done automatically dependent on the mean and standard deviation of every area among four sub regions.

2.1. Histogram Method. This technique is used to convert the input image into a gray image. A histogram equalization technique has been connected to this grayscale image to enhance the intensity image contract, which aids to recognize the brightest portion of the image. Before applying adaptive thresholding with ostu value 0.31 , we shift just over white pixels into full white and others into dark ones. The image is converted to binary after that, morphological dilation and erosion with basic component of $[1 ; 1 ; 1]$ that has better segmentation was implemented at that point. The pixels less than 250 pixels have been expelled as this precisely portioned the districts of the tumor. At long last tumor area is recognized. The histogram strategy for ordering a pixel-by-pixel image characterizes single or multiple thresholds. A fundamental way to determining the threshold value $T$ is by studying the histogram for maximum values and fining the smallest point, typically between two consecutive maximum values of histograms. The statistics method will provide a good result, when a histogram is bi-modal. By equating the gray value of each pixel with the specified threshold $T$, a pixel can be classified into one or two classes. An image $f(x, y)$ may be split into two classes by a gray value limit $T$.

$$
g(x, y)= \begin{cases}1, & \text { if } f(x, y)>T \\ 0, & \text { if } f(x, y) \leq T\end{cases}
$$

Here $g(x, y)$ is the segmented image with two binary values " 1 " and " 0 " and $T$ is the threshold assigned to the smallest point between two histogram peak values.

2.2. Adaptive Threshold. Thresholding is entitled adaptive since a different type of threshold is used for different regions in the image. Thresholding expect that the image has pixel values generally different from the background. This technique approves the threshold value $T$ to alternate depending on the image's progressively different function characteristics. Threshold $T$ relies on the coordinated spatial $(x, y)$ itself. This technique is adapted with some optimization. Following is the outline of adaptive thresholding:

1. Binarizing the image with a single threshold $T$;

2. Thinning the threshold image;

3. Remove all branch points in the thin image;

4. All remaining endpoints are located in the analysis queue and used as an initial point for tracking;

5. Track the region with threshold $T$

6. If the region has passed, $T=T-1$, go to 5

The adaptive thresholding algorithm used a recursive filter to determine the nearby weighted mean in the image just along the row, or set of rows. Here, to calculate the local mean, we use symmetrical 2D Gaussian 


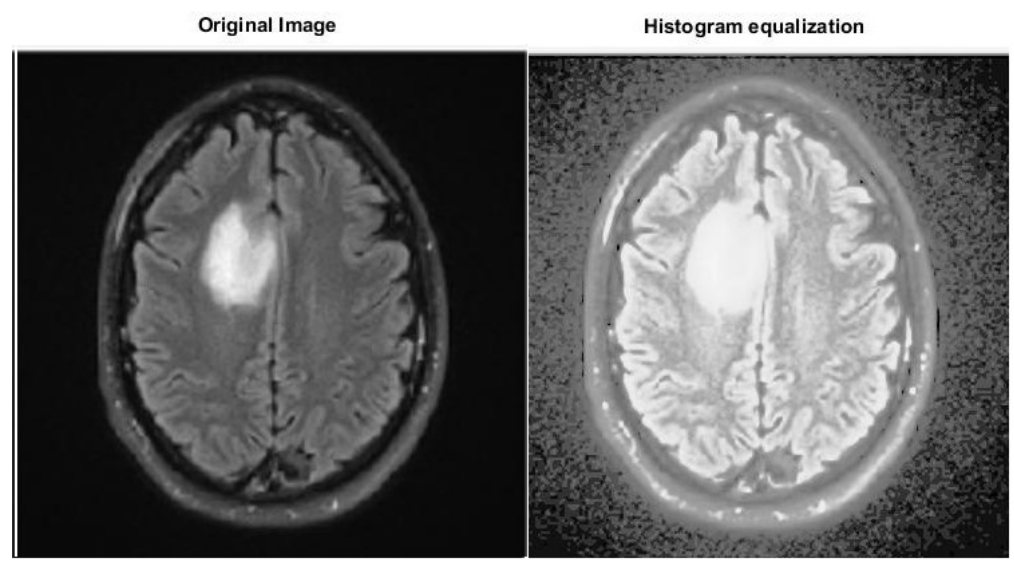

FIG. 2.1. Histogram Equilization with White Pixels

smoothing. This is slower yet gradually wide. Median filtration is an alternative to the mean and offers the option to use a fixed threshold relative to the mean / median.

Let $f s(n)$ is the sum of the $s$ pixel values at point $n$.

The resulting $T(n)$ is either 1 or 0 depending on whether it is $t$ percent darker than the previous $s$ pixels average value.

$$
g(x, y)= \begin{cases}1, & \text { if } P_{n}<\left(\frac{f_{s}(n)}{s}\right)\left(\frac{100-t}{100}\right) \\ 0, & \text { otherwise }\end{cases}
$$

2.3. Anisotropic Diffusion filtering. In order to lighten the noise effects, noise reduction is frequently used before segmentation, characterization and recognition to expel or lessen the noise. Noise reduction is an important approach for image processing that has broad implementation in various areas $[7,8]$. The key is to decrease the noise without breaking down the vital highlights in the image. In this way, noise decrease has two objectives. One is to expel the noise from the image, and the other is to save the imperative highlights, for example, the edges in the image. A uproarious image can frequently be shown as one of the two models depending on the type of noise: a linear model and a nonlinear model. A MR image is commonly demonstrated as a noise model.

The anisotropic diffusion filtering is a general scale space way to deal with edge detection presented by Perona and Malik. In their work they moved from the linear scale-space model which considers the filtered image $I(x, y, t)$ and unique image $I o(x, y)$ implanted in a novel group of functions characterized by

$$
I(x, y, t)=I o(x, y) \cdot G(x, y, t)
$$

The simple equation of anisotropic diffusion as presented in [5] is

$$
\frac{\partial I(x, y, t)}{\partial t}=\operatorname{div}[g(|\Delta I(x, y, t)|) \Delta I(x, y, t)]
$$

where $t$ is the time parameter, the original image is $I(x, y, 0)$, the gradient of the image version at the time $t$, and the so-called conductivity function is $g$.

2.4. Level set method. Interested object's rough boundaries are divided by thresholding method. The extracted portion is regarded as a level-set method initialization [9]. Methods of level-sets rely on partial differential equation to the surfaces of model deformation. Level sets techniques rely on two key embedding techniques; first, the implantation of the surface as the zero level set of a greater velocity to this greater point set feature. A level set equation represents the assessment of the shape or surface. The solution to which this 
partial differential equation tends is calculated iteratively by updating at each interval of moment, below is shown the overall form of the level set equation:

$$
\frac{\partial \varphi}{\partial t}=-|\Delta \varphi| \cdot F
$$

Here, $F$ is the frequency that defines the assessment of the level set. By using $F$, given a specific initialization of the level set function, we can guide the level set to the different areas or shapes. It is also essential to have an initial mask for the level set function, which can take the form of a two-dimensional square or any other closed form. In this article, threshold findings act as a seed for a level-set method and the final tumor region is acquired after a few iterations.

2.5. Feed Forward Artificial Neural network. Feed forward Artificial Neural Network One of the simplest feed forward neural networks (FFNN, consists of three layers: an input layer, hidden layer and output layer. In each layer there are one or more Processing Elements (PEs). PEs is meant to simulate the neurons in the brain and this is why they are often referred to as neurons or nodes. PE receives inputs from either the outside world or the previous layer. There are connections between the PEs in each layer that have a weight (parameter) associated with them. This weight is adjusted during training. Information only travels in the forward direction through the network -there are no feedback loops.

\section{Results and discussion.}

3.1. Performance evaluation. Table 3.1 gives the details of the tumor are present along with the location of it in the brain according the hemispheres of the brain.

The following performance metrics are used to evaluate the efficiency of the proposed algorithm.

True Positive $(T P)=$ the no. of images correctly identified as tumor True Negative $(T N)=$ the no. of images correctly identified as healthy False Negative $(F N)=$ the no. of images incorrectly identified as healthy. False Positive $(F P)=$ the no. of images incorrectly identified as tumor.

TABLE 3.1

Tumor segmentation and its location

\begin{tabular}{|c|c|c|c|c|c|}
\hline Input Image & Histogram Equalization & Tumor Outline & Brain Area (B) & Tumor Area(T) & $\overline{\operatorname{Ratio}(\mathrm{B} / \mathrm{T})}$ \\
\hline & & & 12046 & 114 & 0.946 \\
\hline & & & 24283 & 1435 & 5.90 \\
\hline & & & 1523 & 98 & 0.639 \\
\hline & & 8 & 21652 & 1142 & 5.274 \\
\hline & & & 32970 & 848 & 2.572 \\
\hline & 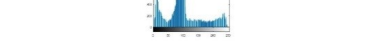 & & 126793 & 3368 & 12.570 \\
\hline
\end{tabular}




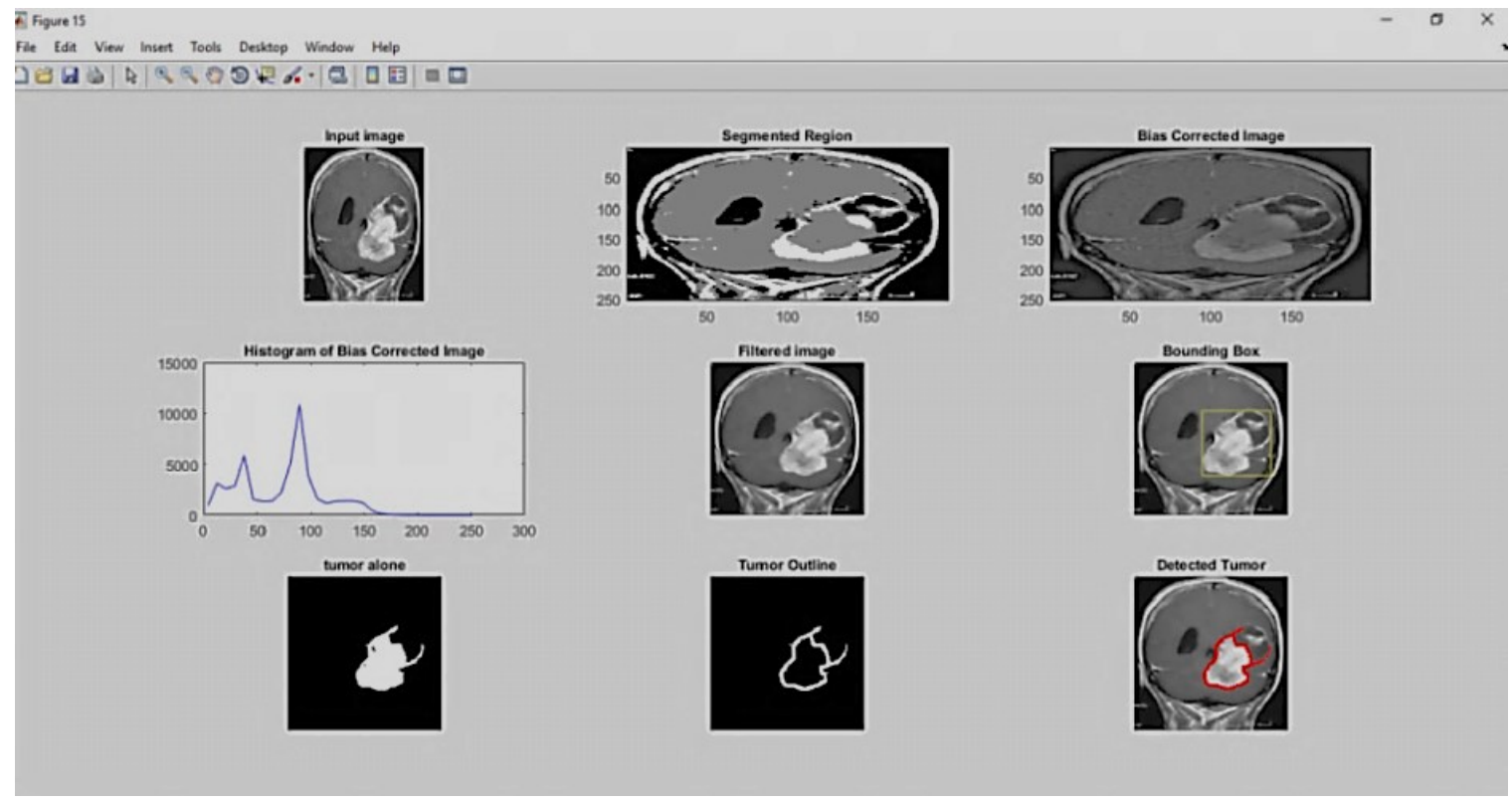

FIG. 3.1. Tumor detection using level set and bounding box with bias corrected image

Accuracy: It is a measure of the closeness of the measurements to true value

$$
\text { Accuracy }=\frac{\sum(T P+T N)}{\sum(T P+T N+F N+F P)} \times 100
$$

Precision: It is definite as the accuracy as the combination of both actuality and exactness

$$
\text { Precision }=\frac{\sum(T P)}{\sum(T P+F P)} \times 100
$$

3.2. Results. In this module, the suggested structure is the detection of tumor slices in which the boundary box is situated around the tumor and acts as a seed for brain tumor segmentation (Figure 3.1). The algorithm set threshold and level set is used to obtain the accurate tumor.

Table 3.2 shows the segmentation of the image with delta value is 0.1429 and different threshold value for the different images.

TABLE 3.2

Performance of brain tumour segmentation based on three evaluation indexes

\begin{tabular}{ccc}
\hline Subject & Density & Threshold \\
\hline P1 & 0.0745 & 0.3725 \\
P2 & 0.0487 & 0.2196 \\
P3 & 0.7941 & 0.2588 \\
P4 & 0.0961 & 0.2471 \\
$:$ & $:$ & $:$ \\
$:$ & $:$ & $:$ \\
$:$ & $:$ & $:$ \\
$:$ & $:$ & $:$ \\
P212 & 0.1669 & 0.1569 \\
\hline Mean & 0.2869 & 0.2492 \\
Std & 0.0141 & 0.0123 \\
\hline
\end{tabular}


TABLE 3.3

Performance of brain tumour segmentation

\begin{tabular}{ccccccc}
\hline Subject & $T P$ & $T N$ & $F P$ & $F N$ & Precision & Recall \\
\hline P1 & 1 & 0 & 0 & 0 & 1 & 1 \\
P2 & 1 & 0 & 0 & 0 & 1 & 1 \\
P3 & 1 & 0 & 0 & 0 & 1 & 1 \\
P4 & 1 & 0 & 0 & 0 & 1 & 1 \\
$:$ & $:$ & $:$ & $:$ & $:$ & $:$ & $:$ \\
$:$ & $:$ & $:$ & $:$ & $:$ & $:$ & $:$ \\
$:$ & $:$ & $:$ & $:$ & $:$ & $:$ & $:$ \\
$:$ & $:$ & $:$ & $:$ & $:$ & $:$ & $:$ \\
P212 & 1 & 0 & 0 & 0 & 1 & 1 \\
\hline Total 212 & 182 & 25 & 0 & 5 & 99.52 & 98.87 \\
\hline
\end{tabular}

TABLE 3.4

Performance of the proposed system

\begin{tabular}{cccccccc}
\hline Total Number of Images & $T P$ & $T N$ & $F P$ & $F N$ & Sensitivity & Specificity & Accuracy \\
\hline 212 & 182 & 25 & 0 & 5 & 97.76 & 100 & 97.32 \\
\hline
\end{tabular}

Of the 187 images taken with the tumor, 182 images were evaluated by the framework efficiently. Other 5 images are incorrectly acknowledged without a tumor. The reason for this failure was that there is no clear difference in pixel intensity between the tumor region and the rest of the brain. The framework correctly recognized 25 images without a tumor as images without a 100 percent successful tumor. The outcome of the suggested framework is shown in Table 3.4.

As discussed above, segmentation of brain tumour plays an important role in diagnostic procedures. The accurate segmentation helps in clinical diagnostic, but also helps to increase the lifetime of the patient. Based on the conventional histogram equalization algorithm, the paper presented an adaptive gray level mapping algorithm which takes the entropy and visual effects as the target function. The selection rule of parameter in different conditions and the identification method of the image gray type are also presented. The result shows that the implemented method helps in detection of enhancing tumour as well as specifying tumour to the actual tumour region only.

4. Conclusion. In identifying tumor tissues in the medicine sector, this paper suggested an outstanding and innovative classification of brain image. The paper provided an adaptive gray level mapping algorithm based on the histogram equalization algorithm that takes entropy and visual effects as the target function. The enhanced images are reconfigured using histogram equalization to reconfigure their pixel levels in histogram techniques. Developing more suitable techniques for detecting malignant brain tumors that are tiny in size would be excellent. The real tumor size can also be calculated from the 3D image.

\section{REFERENCES}

[1] Mouli Laha, Prasun Chandra Tripathi, And Soumen Bag, A Skull stripping from brain MRI Using Adaptive Iterative thresholding and Mathematical Morphology,recent advances in Information Technology RAIT-2018.

[2] K Sudharani, T.C. SARma And K Satya Prasad , Histogram related threshold techniques for region based automatic brain tumor detection, Indian journal of science and technology, dec-2016.

[3] Sudipta Roy, Bhattacharyya D, Samir kumar And Tainoon Kim, An iterative implementation of level set for precise segmentation of brain tissues and abnormality detection from MR Images,IETE Journal of Research, June 2017.

[4] Christopher Bowles, Chen Qin, Ricardo Guerrero, Roger Gunn, And David Alexander Dickie, Brain lesion segmentation through image synthesis and outlier detection, Neuro Image: clinical Elsevier 2017.

[5] Zhang, R., Shen, J., Wei, F., Li, X., And SAngaiah, A. K, LU-Medical image classification based on multi-scale nonnegative sparse coding, Artif. Intell. Med., 2017. https://doi.org/10.1016/j.artmed.2017. 05.006 
[6] Chourmouzios Tsiotsios and Maria Petrou, On the choice the parameter for anisotropic diffusion in image processing, Pattern recognition in Elsevier, 2012.

[7] G. Gerig, O. Kubler, R. Kikinis, And F. A. Jolesz, Nonlinear anisotropic filtering of MRI data, IEEE Transactions on Medical Imaging, vol. 11, no. 2, pp. 221-232, 1992.

[8] J. Tang, S. Millington, S. T. Acton, J. Crandall, and S. Hurwitz, Surface extraction and thickness measurement of the articular cartilage from MR images using directional gradient vector flow snakes, IEEE Transactions on Biomedical Engineering, vol. 53, no. 5, pp. 896-907, 2006.

[9] C. Li. R. Huang, Z. Ding, J. Chris Gatenby, N. Dimtris Metaxas, A level set method for Image segmentation in the Presence of Intensity in homogeneities with application to MRI, IEEE transactions on image processing, vol.20, No. 7, July 2011.

[10] M.H.O. Rashid, M.A Mamun, M.A Hossain and M.P Uddin, Brain tumor detection using Anisotropic Filtering SVM Classifier and Morphologival Operation from MR Images, International conference on Computer, Communication, Chemical, Materials and Electronics Engineering, Feb 2018.

[11] H.R. Shahdoosti, A. Mehrabi, MRI and PET image fusion using structure tensor and dual ripplet-II transform, Multimed. Tools Appl. 77 (17) (2017)22649-22670.

[12] G. Litjens Et Al., A survey on deep learning in medical image analysis, Med. Image Anal., vol. 42, pp. 60-88, Dec. 2017.

[13] X. Zhao, Y. Wu, G. Song, Z. Li, Y. Zhang, and Y. Fan, A deep learning model integrating FCNNs and CRFs for brain tumor segmentation,Med. Image Anal., vol. 43, pp. 98-111, Jan. 2017.

[14] M. Havaei Et Al., Brain tumor segmentation with deep neural networks, Med. Image Anal., vol. 35, pp. 18-31, Jan. 2017.

[15] S. Pereira, A. Pinto, V. Alves, And C. A. Silva, Brain tumor segmentation using convolutional neural networks in MRI images,IEEE Trans.Med. Image vol. 35, no. 5, pp. 1240-1251, May 2016

Edited by: Swaminathan JN

Received: Sep 3, 2019

Accepted: Nov 30, 2019 